Voix et Images

voixetimages

\title{
La littérature? Quelle littérature?
}

Jacques Pelletier

Volume 11, numéro 3 (33), printemps 1986

Yolande Villemaire

URI : https://id.erudit.org/iderudit/200587ar

DOI : https://doi.org/10.7202/200587ar

Aller au sommaire du numéro

\section{Éditeur(s)}

Université du Québec à Montréal

\section{ISSN}

0318-9201 (imprimé)

1705-933X (numérique)

Découvrir la revue

\section{Citer cet article}

Pelletier, J. (1986). La littérature? Quelle littérature? Voix et Images, 11(3),

538-542. https://doi.org/10.7202/200587ar d'utilisation que vous pouvez consulter en ligne.

https://apropos.erudit.org/fr/usagers/politique-dutilisation/ 


\title{
Essai
}

\section{La littérature? Quelle littérature?}

\author{
par Jacques Pelletier, Université du Québec à Montréal
}

Le discours sur la littérature n'est jamais innocent. Toujours il est effectué à partir d'un lieu bien précis et dans l'optique privilégiée par ce lieu. Si quelqu'un en doutait encore, je lui en fournirais comme preuves les ouvrages que viennent de faire paraître récemment François Ricard ${ }^{1}$ et Maurice Arguin ${ }^{2}$.

Le premier auteur, on le sait, est un personnage public: professeur, animateur à la radio et à la télévision, il était jusqu'à récemment directeur de la revue Liberté. Cette revue, malgré ce qu'en disent ses rédacteurs dont la modestie souvent proclamée ne doit pas nous leurrer, est un lieu de pouvoir important de l'institution littéraire québécoise, les membres de son comité de direction et ses collaborateurs occupant des positions dominantes à l'Université (U.D.M., U.Q.A.M., McGill), dans les journaux (Le Devoir), la radio (secteur culturel et littéraire de Radio-Canada), les jurys (à Québec et à Ottawa), le cinéma (l'O.N.F.), l'édition (le Boréal Express où Godbout et Ricard occupent des fonctions stratégiques) etc. Or cette revue défend une certaine conception de la littérature qui constitue en quelque sorte l'équivalent, dans le champ littéraire, de la conception de la langue entretenue par Radio-Canada (promotion du "français international" par opposition au français québécois): défense en l'occurrence de la "grande littérature internationale (et à l'occasion nationale)" et rejet des productions de masse (littérature dégradée) d'une part et d'avant-garde (excès de sophistication pour "happy few») d'autre part.

Le livre de Ricard s'inscrit tout naturellement dans cette perspective, l'auteur se nourrissant en effet-d'un idéal de littérature mondiale (p. 190) et dialoguant en quelque sorte avec les "grands" de cette littérature, abstraction faite de leur lieu d'appartenance. À ce titre il est significatif que son livre soit introduit par une préface de Milan Kundera, caution précieuse à la fois de la qualité de la marchandise présentée et du niveau-international-du discours tenu: Ricard, écrit le célèbre auteur tchèque, habite la littérature mondiale et, comme il s'occupe presque exclusivement du roman, je peux dire qu'il habite le roman mondial comme on habite une maison (p. 10).

Fort de cet éloge décerné par un écrivain prestigieux dont le capital symbolique est singulièrement élevé par les temps qui courent, l'auteur présente ensuite modestement son ouvrage comme un livre fragmentaire et circonspect. 
Fragmentaire car constitué de textes déjà parus pour l'essentiel dans Liberté. Circonspect car relevant d'une attitude de perplexité et de méfiance par rapport au discours critique et à la littérature elle-même. Pas d'ambition théorique et/ou méthodologique donc à la base de l'ouvrage mais plutôt une volonté d'écoute attentive, un peu à la manière de Bachelard, laissant les textes résonner en soi et provoquer cet ébranlement, cette vibration mentale que créent pour moi certaines auvres et certains écrivains (p. 13). Position qui est elle-même le terme - provisoire sans doute - d'un itinéraire ayant conduit l'auteur de la foi en la littérature comme «instrument de connaissance » à un certain agnosticisme refusant de voir en celle-ci une voie quelconque de salut (p. 18). Mais tout compte fait, cela n'empêche pas, malgré tout, que demeure paradoxalement l'amour de la littérature la seule chose qui, me trompant, avoue en même temps sa tromperie(p. 21).

Ceci étant, que nous dit Ricard sur les textes qui lui parlent? Et en quoi son propos peut-il nous intéresser?

Je dirai d'abord que ses analyses - et c'est à mes yeux un mérite surprennent dans la mesure où elles s'inscrivent en faux par rapport à bien des idées reçues. À titre d'exemple il écrira à propos de l'œuvre de son préfacier qu'elle n'est pas d'abord une simple expression de ce qu'on a convenu d'appeler la dissidence et qu'une lecture historico-idéologique simple des romans de Kundera ne peut être qu'une récupération (p. 25). Leur vérité profonde est ailleurs, dans un refus non seulement d'un type de régime politique précis, mais de la "réalité" politique elle-même: La subversion politique, ici, est globale, écrit Ricard. Elle ne s'attaque pas seulement à telle ou telle réalisation mais bien à l'idée même, à l'idole (dirait Valéry, à qui Kundera ressemble par plus d'un côté) de la politique (p. 26). Remarque intéressante qui ne manque pas de pertinence, on le voit, mais qui pose toutefois problème. Car comment ne pas voir que ce refus radical de la politique est lié justement à une expérience concrète, qu'il prend racine dans le contexte des régimes bureaucratiques des pays de l'Est et que ce refus, par conséquent, aussi absolu et en quelque sorte "métaphysique" qu'il soit, possède une histoire, une genèse qui en rend possible l'émergence?

En bref, si l'observation de Ricard est juste, elle est en même temps limitée dans la mesure même où elle refuse de prendre en compte l'histoire (qui fait pourtant l'objet d'un éloge ailleurs dans son livre - j'y reviendrai).

J'évoquerai comme autre exemple l'analyse d'un auteur québécois cette fois, André Major - le seul à être objet d'étude avec Gilles Archambault (autre membre du réseau Radio-Canada - Liberté, réseau, je le signale en passant, qu'il serait très instructif et révélateur de décrire dans la perspective institutionnelle prônée par l'école de Bourdieu) et Gabrielle Roy. Des Histoires de déserteurs de Major, Ricard écrit qu'elles marquent un tournant, une étape majeure aussi bien dans l'évolution littéraire de leur auteur que dans l'évolution récente du roman québécois (p. 57). Et plus loin il précise que ce tournant est comparable à celui marqué par l'œuvre d'un Laberge au début du siècle ou à celui de Bonheur d'occasion au milieu des années quarante (p. 63). 
Bon. Encore là on retrouve d'une part une observation juste, perspicace: la trilogie de Major marque incontestablement un tournant dans l'œuvre de cet auteur. Mais doit-on pour autant partager les corollaires qui, pour Ricard, découlent de cette observation, à savoir, premièrement que les cuvres antérieures de l'écrivain sont des écrits préparatoires, une production de jeunesse qui ne vaut que comme amorce, esquisse de la trilogie qui, dans cette perspective, apparaît comme un couronnement et deuxièmement què cette production marque un tournant dans l'histoire du roman québécois contemporain? Pour ma part j'estime que ces deux corollaires sont très discutables et qu'on peut tout aussi bien penser que les œuvres de jeunesse de Major, si elles ne sont peut-être pas aussi «réussies» techniquement que celles qui forment la trilogie, n'en constituent pas moins des réalisations remarquables sur un plan littéraire plus général et que par ailleurs leur signification historique et littéraire, si l'on ne dissocie pas ces deux plans, contrairement à ce qu'a tendance à faire ici Ricard, est tout au moins aussi importante que les Histoires de déserteurs. Donc à nouveau, à mon sens, comme dans l'analyse des œuvres de Kundera, une observation juste au départ qui est de nature à susciter la réflexion - c'est sa qualité indéniable - mais qui n'entraîne pas forcément l'adhésion (du moins pas la mienne!) dans la mesure où elle a tendance à minimiser l'importance de la dimension historique des productions considérées.

Or, paradoxalement, Ricard aime et pratique l'histoire. Et sa réflexion sur l'historiographie ne manque pas d'à-propos. Il est tout à fait conscient que l'histoire, en un sens, n'existe pas dans la mesure où le passé est du temps mort dont seules des marques, des traces actuelles témoignent d'une existence à jamais révolue si bien que l'historiographie est d'abord un discours écrit par des contemporains, pour des contemporains, création rhétorique d'un passé en fonction des attentes et des partialités du présent (p. 149). Tout cela (et les exemples évoqués à l'appui de la réflexion théorique) m'apparaît très juste. Et, au fond, le paradoxe que je signalais plus haut n'est peut-être pas aussi absurde que je le croyais dans la mesure où les deux discours (celui de la littérature, celui de l'histoire) relèvent de conceptions différentes et se voient attribuer par conséquent un statut différent.

Si le discours critique de Ricard porte indiscutablement la marque de Liberté, celui d'Arguin porte pour sa part la griffe de ce que j'ai bien envie d'appeler l'école nationaliste, pour ne pas dire nationaleuse, de Québec. Elle gravite autour de la revue Québec français, du Dictionnaire des ceuvres littéraires du Québec et du nouveau centre de recherche en littérature québécoise de l'Université Laval, dont cet ouvrage constitue la première publication. Contrairement à Liberté qui privilégie le texte international, ici on valorise à l'excès ce que Godbout appelait naguère le texte national, cultivant non sans une complaisance certaine le culte de la québécitude, cette conception mythique et mystifiante de l'idéntité québécoise.

L'ouvrage d'Arguin n'échappe pas à cette perspective, y compris au niveau de certains tics d'écriture, dont à titre d'exemple le recours obsédant à des expressions comme l'homme d'ici défini bien sûr par l'aliénation, la dépos- 
session etc... Cependant on ne saurait honnêtement le réduire à une misérable mise en forme de ces tics, le livre demeurant intéressant par certains aspects en dépit de sa problématique nationaliste étroite et quelque peu datée, c'est le moins qu'on puisse dire.

L'analyse d'Arguin repose en effet sur l'hypothèse que le Québec constitue une société colonisée et que la production romanesque de cette société doit par conséquent être lue et interprétée à la lumière de cette donnée centrale. Cette problématique on le sait, a été élaborée par les animateurs de Parti pris au début des années 1960 , eux-mêmes influencés par les écrits des théoriciens de la décolonisation: Berque, Fanon, Memmi.

Son ouvrage comporte trois parties dont les deux premières sont consacrées à la production romanesque des années 1940 et 1950 . Dans ce corpus, l'auteur s'attache plus spécifiquement à décrire comment la situation d'aliénation du Canadien français est représentée dans le roman de mours urbaines et dans le roman dit psychologique. Dans la troisième partie il montre que le roman du début des années 1960 témoigne d'une prise de conscience aiguë de cette aliénation et d'une volonté résolue d'y mettre fin.

Le postulat théorique d'Arguin accepté, l'analyse qui en découle est généralement cohérente et convaincante. Mais pas toujours. Ainsi, étudiant le roman urbain, s'il a raison de souligner que Montréal n'est pas divisé seülement sur le plan géographique et social mais aussi ethnique, il a tort à mon sens de privilégier cette dimension au détriment des autres et de revendiquer comme caution de son analyse la conception du Canada français comme classe ethnique développée par Marcel Rioux au début des années 1960 , cette conception reléguant à l'insignifiance les divisions inter-classistes bien réelles existant au sein de cette société. J'en veux comme preuve des méprises auxquelles peut conduire une analyse guidée par cette problématique l'étude qu'Arguin soumet d'Au pied de la pente douce de Lemelin, roman dont certains passages, à l'en croire, préfigurent presque mot à mot, le Portrait du colonisé (p. 71) - rien de moins! Et cela dans une ville quasi homogène sur le plan ethnique, qui ne ressent guère, c'est le moins qu'on puisse dire, le poids de «l'occupant»!

Par ailleurs, étudiant le roman psychologique, il s'appliquera à faire ressortir que les problèmes individuels des héros de ces romans sont aussi collectifs, et qu'à ce niveau encore une fois c'est le rapport colonial qui est décisif. Bien entendu, de telles observations contiennent leur part de vérité mais au détriment de la prise en compte d'un phénomème structurel, à mon sens, plus déterminant qui est le passage de la société québécoise d'un cadre de référence traditionnel à l'univers éclaté généré par sa modernisation. Ce roman met essentiellement en lumière la crise de valeurs créée par cette transformation. Et ce n'est pas un hasard s'il s'est imposé comme la forme romanesque privilégiée par la petite bourgeoisie intellectuelle de la période qui s'y reconnaissait comme l'a bien montré Jacques Michon dans une étude remarquable ${ }^{3}$ qu'Arguin aurait eu intérêt à connaître, ce qui lui aurait permis de raffiner ses analyses. De même, et bien que cela soit moins important, il 
minimise l'influence qu'a pu exercer sur cette production le roman existentialiste français. Quand on sait combien notre production littéraire de cette période dépendait encore largement de la tradition littéraire française, une telle position qui secondarise (trop à mon avis) les effets de champ n'est pas sans susciter de l'étonnement.

Ceci étant, une fois la problématique de la décolonisation admise, il faut reconnaître quẹ. les études d'Arguin sont généralement bien menées. Mais c'est justement là où le bât blesse. Car la problématique élaborée à l'époque de Parti pris et qui est un produit de cette époque est fortement remise en question aujourd'hui aussi bien par ceux qui la soutenaient alors que par de nombreux critiques (marxistes comme non marxistes) de la société québécoise. Or Arguin utilise cette problématique comme un postulat, une sorte d'évidence, sans s'interroger sur sa pertinence. Mais cela ne va pas de soi. Bien au contraire tout semble démontrer aujourd hui que la théorie du «socialisme décolonisateur» a fait son temps, que la "grille coloniale" n'explique plus grand chose et qu'elle doit elle-même être remise dans son contexte et expliquée. C'est là il me semble la faille majeure de ce travail dont les analyses de détail sont le plus souvent justes et pertinentes malgré un cadre général d'interprétation qui, lui, ne résiste pas à l'examen.

C'est ainsi que, chacun à leur manière, les ouvrages d'Arguin et de Ricard révèlent combien le discours d'accompagnement de la critique peut servir d'aune pour délimiter ce qui, dans l'ensemble des textes écrits, doit être privilégié comme littérature signifiante. Et ce discours est lui-même tributaire du lieu où il est énoncé, qu'il s'agisse d'un Montréal branché sur l'Europe ou d'un Québec fixé sur "la littérature d'ici»; dans tous les cas le "d'où je parle» détermine «ce dont je vais parler».

1. François Ricard, la Littérature contre elle-même, Montréal, Boréal Express, coll. Papiers Collés, 1985. 195 p.

2. Maurice Arguin, le Roman québécois de 1944 à 1965. Symptômes du colonialisme et signes de libération, Québec, C.R.E.L.I.Q., coll. Essais, 1985, 227 p.

3. Jacques Michon «Esthétique et réception du roman conforme, 1940-1957", in Structure, idéologie et réception du roman québécois de 1940 à 1960. Sherbrooke, Département d'études françaises, Université de Sherbrooke, coll. Cahiers d'études littéraires et culturelles. 1979, p. 4-20. 